\title{
Sequential modulations of correspondence effects across spatial dimensions and tasks
}

\author{
WILFRIED KUNDE \\ Martin-Luther-Universität, Halle-Wittenberg, Germany \\ and \\ PETER WÜHR \\ Friedrich-Alexander-Universität, Erlangen, Germany
}

\begin{abstract}
In two experiments, we explored sequential modulations of correspondence effects in a prime-target paradigm. In Experiment 1, the participants responded to the direction of target arrows that were preceded by prime arrows with a corresponding or noncorresponding direction. This produced a prime-target correspondence effect that was reduced when the preceding trial contained a noncorresponding prime-target event. This sequential modulation of the correspondence effect was observed even when neither stimuli nor responses were repeated from one trial to the next, ruling out explanations of sequential modulations in terms of stimulus or response repetitions. Experiment 2 combined the prime-target correspondence effect with a Simon-type correspondence effect. Both effects were reduced following noncorrespondence of the same type and, to a lesser extent, following noncorrespondence of the other type. Altogether, these results suggest that part of the sequential modulation of correspondence effects reflects an adaptation to a preceding response conflict independently of the peripheral stimulus events that produced this conflict.
\end{abstract}

Many of our everyday activities are subserved by highly practiced stimulus-response (S-R) routines. Pushing a car's gas pedal at a green traffic light, grasping for a cup of coffee, or shaking hands with someone at a meeting are examples of cases in which we encounter situations with approved, and thus prepotent, behavioral responses. Sometimes, however, routine responses are inadequate. For example, we must not press the gas pedal when the green traffic light signals another lane, and grasping a cup in the standard way may be dangerous if the cup has an atypical shape. Thus, habitual responses to a given situation can turn out to be inapplicable. These are situations in which cognitive control is assumed to come into play (e.g., Norman \& Shallice, 1986). A natural consequence of experiencing such problems is to abandon behavioral routines and to enter into a more cautious response mode.

With so-called conflict tasks, it is possible to study experimentally how our cognitive system handles such problems. In these tasks, a conflict can occur between a certain instructed response to a stimulus and another, prepotent response suggested by some nominally task-irrelevant aspect of the stimulus display. Typically, performance

\footnotetext{
Funding for this research was provided by the German Research Foundation to Joachim Hoffmann and W.K. (Ho 1301, Ku 1964). We thank Colin MacLeod, Ulrich Mayr, and Robert Proctor for helpful comments on a previous version of the manuscript. Thanks also to Henrike Krauß for collecting the data and Sarah Mannion for improving the English. Correspondence concerning this article should be sent to W. Kunde, Institut für Psychologie, Martin-Luther-Universität Halle-Wittenberg, D 06099 Halle (Saale), Germany (e-mail: w.kunde@psych.uni-halle.de or prwuehr@phil.uni-erlangen.de).
}

deteriorates when such a conflict exists, as compared with when it does not. For example, in the Simon task, responding is slower and more error prone when the taskirrelevant location of a stimulus and the location of the stimulus-assigned response do not match (e.g., responding to a left-sided stimulus with a right-sided response) than when they do match (Simon, 1969). Another conflict task is the Stroop task, in which participants are required to name the color of color words (e.g., the word red presented in green color). In other types of conflict tasks, the irrelevant information is conveyed by an extra stimulus, as in the prime-target paradigm. Here, task-relevant targets (e.g., a left-pointing arrow calling for a left keypress) are preceded by irrelevant but target-resembling primes (e.g., another arrow). Again, performance is superior when the prime and the target indicate identical, rather than different, responses (e.g., Neumann \& Klotz, 1994; Vorberg, Mattler, Heinecke, Schmidt, \& Schwarzbach, 2003).

An interesting question relates to the processes that come into play after a response conflict has occurred. One robust finding at the behavioral level is that many compatibility effects are reduced after an incompatible $\mathrm{S}-\mathrm{R}$ episode. This is true for different conflict tasks, such as the Simon task (Hommel, Proctor, \& Vu, 2004; Kunde \& Stöcker, 2002; Praamstra, Kleine, \& Schnitzler, 1999; Stürmer, Leuthold, Soetens, Schröter, \& Sommer, 2002), the Eriksen task (Botvinick, Nystrom, Fissell, Carter, \& Cohen, 1999; Gratton, Coles, \& Donchin, 1992), and the prime-target task (Kunde, 2003).

Basically, two different accounts of such sequential modulations have been offered. The gating account attributes sequential effects to increased cognitive control 
after the occurrence of a response conflict. The idea is that observers are able to allow or disallow stimulus-driven activation of prepotent responses (e.g., Stürmer et al., 2002). If this activation has turned out to be helpful (after a compatible trial), the activation is allowed for subsequent trials. This produces great benefits for a subsequent compatible S-R event and great costs for a subsequent incompatible S-R event-hence, an overall strong compatibility effect in the following trial. If, however, stimulus-driven activation of prepotent responses has proven to be detrimental (after incompatible trials), the system focuses on relevant stimulus aspects and, thereby, reduces the impact of irrelevant stimulus features (Botvinick, Braver, Barch, Carter, $\&$ Cohen, 2001). This reduces the benefits for a subsequent compatible $\mathrm{S}-\mathrm{R}$ event and the costs for a subsequent incompatible S-R event; hence, an overall reduced compatibility effect results. As was noted above, response conflicts can occur for several reasons-for example, because of a mismatch between the spatial positions of a stimulus and the response that it requires (Simon effect) or because other irrelevant stimuli signal a currently unwanted response (Eriksen effect). For the gating account, the critical event for triggering a sequential modulation of correspondence effects is the detection of a response conflict. Less relevant is the peripheral event that caused this conflict (an incompatible stimulus location, flanker, or prime). A favorable aspect of the gating account is, thus, that it qualifies as a broad account for sequential effects in several different conflict tasks.

The repetition/alternation account attributes sequential modulations to an unbalanced proportion of partial stimulus-response repetitions when correspondence effects are analyzed as a function of correspondence in the preceding trial. Normally, responding is faster when the stimuli and the responses are completely repeated or completely alternated than when the stimulus changes but the response is repeated and, thus, a partial repetition of the preceding S-R event occurs (Bertelson \& Renkin, 1966; Hommel et al., 2004; Soetens, 1998). Crucially, in two-choice reaction tasks (2-CRTs), all sequences of corresponding S-R events are either complete repetitions or complete alternations of the $\mathrm{S}-\mathrm{R}$ episode. This might inflate correspondence effects when a corresponding-corresponding $(\mathrm{C}-\mathrm{C})$ sequence is compared with a corresponding-noncorresponding $(\mathrm{C}-\mathrm{NC})$ sequence. Also, all sequences of noncorresponding trials are either complete repetitions or complete alternations. This might reduce the correspondence effects when an $\mathrm{NC}-\mathrm{NC}$ sequence is compared with an $\mathrm{NC}-\mathrm{C}$ sequence. In other words, sequential modulations of correspondence effects might simply be mimicked by complete repetition/ alternation benefits. Obviously, this account would render the study of cognitive control in sequential modulations of correspondence effects meaningless, since these modulations were not a consequence of cognitive control operations at all.

The present article reports two experiments that help to evaluate these competing accounts. In Experiment 1, we assessed sequential modulations of correspondence effects in a prime-target paradigm. We investigated whether sequential effects persist when neither the stimuli nor the responses are repeated from one trial to the next. Such a result would leave the gating account as a viable explanation but would refute the repetition/alternation account, which hinges crucially on the comparison between complete and incomplete repetitions/alternations. We also varied the duration of primes to explore whether the size and/or conspicuity of response conflict has a bearing on sequential effects. Experiment 2 explored the extent to which sequential effects reported in several different paradigms bear on a common mechanism. To test this, we investigated whether sequential effects occur between different types of correspondence effects (i.e., between prime-target correspondence effects and the Simon effect). Again, such a result would be in accordance with the idea of a common response conflict adaptation mechanism, whereas it would not easily be reconciled with an explanation in terms of repetitions/alternations of specific stimuli or responses.

\section{EXPERIMENT 1}

The repetition/alternation account hinges on the fact that the succession of corresponding and noncorresponding trials normally consists, to varying degrees, of incomplete $\mathrm{S}-\mathrm{R}$ repetitions. This problem is insurmountable in 2-CRTs, where the succession of corresponding/ noncorresponding trials cannot be varied independently of the occurrence of complete/incomplete $\mathrm{S}-\mathrm{R}$ repetitions (Hommel et al., 2004). A way of eliminating this problem is to use more than two stimuli (and responses). This makes it possible to analyze trial sequences in which neither the stimuli nor the responses are repeated from one trial to the next.

Interestingly, some recent studies that have followed this approach showed remarkably contradictory results. For example, Kerns et al. (2004) observed sequential modulations in a Stroop task with three colors, with repetitions of colors and color words excluded from the analysis. Also, Wühr (2005) investigated a variant of the Simon task with four stimulus positions and two response positions and observed sequential modulations of the Simon effect even when only complete alternations were considered. By contrast, sequential effects were absent without response repetitions in a four-choice Eriksen task or when, in a twochoice version of the task, only response alternations were considered (Mayr, Awh, \& Laurey, 2003).

Taking into account all of this evidence, it seems likely that sequential effects have several causes, which depend on the particular task employed. It would therefore appear to be unrealistic to ultimately resolve the contradictory evidence for all existing conflict tasks put together. What can be done, however, is to clarify the extent to which sequential effects in a particular task (or a particular combination of tasks) can be construed as reflecting cognitive control operations. This would constitute important information 
for researchers wishing to use such sequential effects as a tool for the study of cognitive control.

To this end, in Experiment 1, we investigated sequential effects in a prime-target paradigm. The task was to respond to the direction of a target arrow that was preceded by a prime arrow with a corresponding or a noncorresponding direction. The stimuli and responses varied on the horizontal and vertical dimensions, respectively. This enabled us to analyze compatibility sequences that resulted from prime-target correspondence on the horizontal dimension in trial $n-1$ and from prime-target correspondence on the vertical dimension in trial $n$, or vice versa. In these cases, the stimuli and responses change completely between subsequent trials, and thus, the repetition/alternation account predicts the absence of sequential modulations of S-R compatibility effects. In contrast, response conflict occurs despite the removal of repetitions, and thus, the gating account predicts that sequential modulations should persist.

In an earlier study in which a two-choice version of the present prime-target task was employed, we had already found sequential modulations of the correspondence effects, which we attributed to control operation following response conflict (Kunde, 2003). This conclusion was supported by the observation that sequential effects were strong when the prime duration was long (126 msec) and the resulting response conflict was large and easily discernible but were weak when the prime duration was short (14 msec) and the conflict was small and less easy to discern (see also Greenwald, Draine, \& Abrams, 1996). This result is in accordance with the idea of conflict adaptation, because such adaptation will be more likely to occur the larger and more obvious such conflict has been. To explore whether this conclusion could be generalized to a four-choice reaction task in which the confound between partial repetitions and type of correspondence sequence could be eliminated, we also varied the presentation duration of the primes in Experiment 1.

\section{Participants \\ Method}

Twenty-four undergraduates ( 8 men and 16 women), 18-31 years of age, participated in the study in fulfillment of a course requirement. Sixteen undergraduates were from the University of Würzburg, and 8 were from the University of Halle.

\section{Apparatus and Stimuli}

An IBM-compatible computer with a 17 -in. VGA display was used for stimulus presentation and response sampling. The stimuli were presented in blue color on a white background. Viewing distance was approximately $80 \mathrm{~cm}$. During the experiment, a central fixation cross $(8 \times 8 \mathrm{~mm})$ was displayed. Primes were arrows of the type depicted in Figure 1 and had a size of $15 \times 10 \mathrm{~mm}$. They pointed left, right, up, or down. Targets were enlarged primes (target/ prime ratio of 2.35). The primes fitted exactly into a cutting in the center of the targets. This strongly affects the perceptibility of the primes with short prime durations - a phenomenon known as metacontrast masking (see Figure 1). The responses were recorded by four microswitches $(12 \times 12 \mathrm{~mm}$ in size $)$ connected to the parallel port of the computer and positioned centrally in front of the participants.

\section{Procedure}

Choice reaction task. Each trial began with an auditory 20-msec warning click $(100 \mathrm{~Hz}) ; 750 \mathrm{msec}$ after click onset, a prime was presented for a randomly determined duration of either 14 or $126 \mathrm{msec}$, followed by a blank interval of $28 \mathrm{msec}$. Next, a target was presented for $126 \mathrm{msec}$. The participants were instructed to respond to the direction of the target with a spatially corresponding key (i.e., a left key for a left-pointing arrow, an up key for an up-pointing arrow, etc.). The layout of the keys and the interkey distances resembled those of the 2, 4, 6, and 8 keys on a numerical pad of a standard $\mathrm{PC}$ keyboard. The left and right keys were pressed with the middle fingers of the left and right hands, respectively. Half of the participants pressed the up key with the left index finger and the down key with the right index finger (S-R Mapping 1), and this mapping was reversed for the other half of the participants (S-R Mapping 2). An incorrect response was fed back to the participants by the message Fehler (error in German), which was displayed for $500 \mathrm{msec}$. The next trial began 1,000 msec after response or the error message.

There were 32 different trial types resulting from the orthogonal combination of 4 prime directions (left, right, up, or down) $\times 4$ target directions (left, right, up, or down $) \times 2$ prime durations $(14$ or

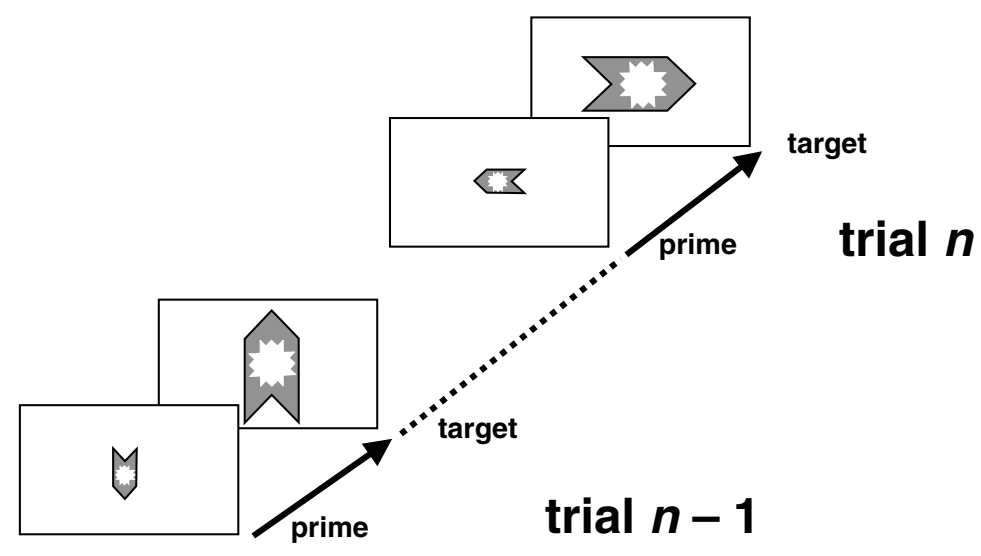

Figure 1. An example of dimension change trial succession in Experiment 1. The prime and the target in trial $n$ were from the horizontal dimension, whereas the prime and the target in trial $n-1$ were from the vertical dimension. 
$126 \mathrm{msec}$ ). The sequential order of trials was arranged so that each of these 32 trial types in trial $n$ was followed once by each of the 32 trial types in trial $n \pm 1$, resulting in a total of 1,024 trials. The participants performed two sessions of 1,024 experimental trials each. These sessions were completed within 1 week. For each participant and session, a new sequential order was computed. Each session began with 24 practice trials. After every 256 trials, a brief break was possible. Following a break, the last 4 trials before the break were repeated as warm-up trials, to allow a meaningful analysis of sequential effects across breaks. These warm-up trials were not recorded.

Prime discrimination task. Following these experimental trials, the participants performed a prime discrimination task. The type and timing of the stimuli in these trials were identical to those in the experimental trials. However, this time the participants were no longer instructed to respond to the targets but were told, instead, to decide at leisure whether the prime presented pointed to the left, the right, up, or down. The participants made these judgments by pressing one of the four 2, 4, 8, or 6 keys on the numerical pad of a PC keyboard in a forced choice situation. For this task, the participants performed 128 trials that resulted from the combination of 4 primes $\times 4$ targets $\times 2$ prime durations $\times 4$ replications. Error feedback was provided at the end of the discrimination task.

\section{Results}

\section{Response Priming}

We removed from our analysis trials with response times (RTs) below $150 \mathrm{msec}$ and above $1,500 \mathrm{msec}(0.05 \%$ of the trials) and trials following errors.

A first analysis looked for potential influences of the mappings of fingers to the response keys (whether the mapping for the index fingers was left-up, right-down [Mapping 1] or left-down, right-up [Mapping 2]). Figure 2 shows RTs as a function of prime direction and target direction for the two S-R mappings employed. It is immediately apparent that there were strong benefits of corresponding primes but no differences among noncorresponding primes, irrespective of the S-R mapping. This is important because one may argue that operating keys with the same hand (e.g., left and up keys with the left hand) could lead to some type of response grouping, so that primes from the horizontal dimension (e.g., left) would also activate a response from the vertical dimension (e.g., up). Clearly this was not the case. This result accords with studies showing that the anatomical connection of response finger to the same hand is not a major factor in the grouping of responses together (Reeve \& Proctor, 1984).

The data were then subjected to an ANOVA with the variables of dimension repetition (whether or not prime or target dimensions [i.e., vertical or horizontal] from the preceding trial were repeated), prime-target correspondence (corresponding or noncorresponding), prime-target correspondence in trial $n-1$ (also corresponding or noncorresponding), prime duration (14 or $126 \mathrm{msec}$ ), and prime duration in trial $n-1$ (also 14 or $126 \mathrm{msec}$ ). Table 1 shows the mean RTs and error rates from the orthogonal combination of these factors. The significance level for this and all the subsequent analyses was set at $5 \%$.

\section{Response Times}

Responding was faster with prime-target correspondence $(M=357 \mathrm{msec})$ than with noncorrespondence $(M=428 \mathrm{msec})[F(1,23)=635.10]$. The correspondence effect was much larger for 126-msec primes $(D=$ $118 \mathrm{msec})$ than for $14-\mathrm{msec}$ primes $(D=26 \mathrm{msec})$, producing an interaction of prime duration and correspondence $[F(1,23)=252.5]$. Responding was particularly fast with 126-msec corresponding primes, resulting in overall slightly faster responses with 126 -msec primes $[F(1,23)=18.47]$.

Of particular interest in the present context were sequential influences from preceding trials. Several such sequential effects were found. First, the correspondence effect was modulated by correspondence in the preced-
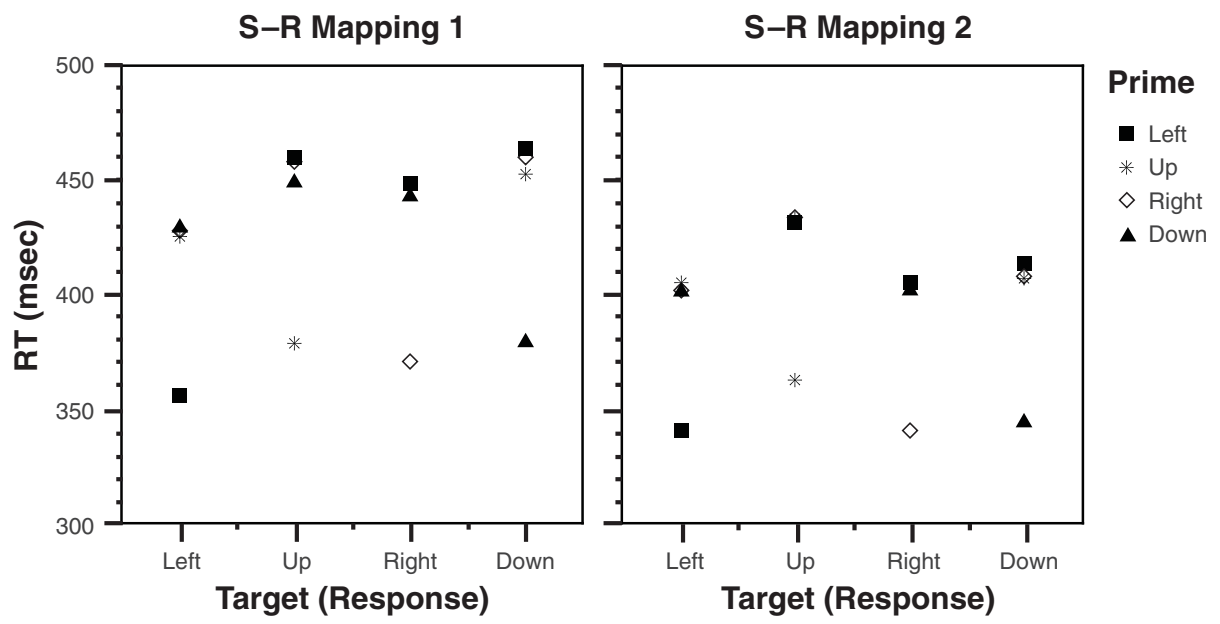

Figure 2. Experiment 1: Response times (RTs) as a function of prime and target (response). The left panel shows the data from the participants responding to upward arrows with the left index finger and to downward arrows with the right index finger. The right panel shows the data from the participants responding to upward arrows with the right index finger and to downward arrows with the left index finger. 
Table 1

Experiment 1: Response Times (RTs, in Milliseconds) and Error Rates

(Percentages of Errors [PEs]) as a Function of Prime Visibility and Correspondence in Trials $n$ and $n-1$ for Dimension Repeat and Dimension Change Trials

\begin{tabular}{|c|c|c|c|c|c|c|c|c|}
\hline \multirow[b]{4}{*}{ Trial $n-1$} & \multicolumn{8}{|c|}{ Trial $n$} \\
\hline & \multicolumn{4}{|c|}{ Less Visible Prime } & \multicolumn{4}{|c|}{ More Visible Prime } \\
\hline & \multicolumn{2}{|c|}{ Noncorresponding } & \multicolumn{2}{|c|}{ Corresponding } & \multicolumn{2}{|c|}{ Noncorresponding } & \multicolumn{2}{|c|}{ Corresponding } \\
\hline & RT & $\mathrm{PE}$ & RT & PE & RT & $\mathrm{PE}$ & RT & PE \\
\hline \multicolumn{9}{|c|}{ Dimension Repeat Trials } \\
\hline \multicolumn{9}{|l|}{ Less visible prime } \\
\hline Noncorresponding & 403 & 2.8 & 380 & 1.9 & 447 & 6.9 & 325 & 1.6 \\
\hline Corresponding & 404 & 2.9 & 371 & 1.0 & 448 & 7.8 & 317 & 1.0 \\
\hline \multicolumn{9}{|l|}{ More visible prime } \\
\hline Noncorresponding & 413 & 2.3 & 391 & 1.9 & 445 & 4.7 & 340 & 1.9 \\
\hline Corresponding & 410 & 2.7 & 375 & 1.1 & 446 & 8.3 & 315 & 1.1 \\
\hline \multicolumn{9}{|c|}{ Dimension Change Trials } \\
\hline \multicolumn{9}{|l|}{ Less visible prime } \\
\hline Noncorresponding & 409 & 1.3 & 387 & 1.6 & 447 & 6.4 & 327 & 1.0 \\
\hline Corresponding & 406 & 3.0 & 383 & 3.5 & 443 & 5.8 & 323 & 1.3 \\
\hline \multicolumn{9}{|l|}{ More visible prime } \\
\hline Noncorresponding & 418 & 1.9 & 401 & 2.0 & 450 & 4.0 & 350 & 0.6 \\
\hline Corresponding & 419 & 1.8 & 388 & 2.3 & 448 & 8.4 & 328 & 1.0 \\
\hline
\end{tabular}

ing trial. It was larger when the prime in the preceding trial was corresponding $(D=78 \mathrm{msec})$, rather than noncorresponding $(D=66 \mathrm{msec}$, rounded) $[F(1,23)=$ 22.52]. Figure 3 shows that this was the case for dimension repeat trials, as well as for dimension change trials $[F(1,23)=1.47, p>.231$, for the interaction of present correspondence, preceding correspondence, and dimension change]. The sequential modulation was significant for dimension repeat trials $(D=14 \mathrm{msec})$, as well as for dimension change trials $(D=11 \mathrm{msec})$ (both $p \mathrm{~s}<.03$, two-tailed $t$ tests). Second, responding was, on the whole, slower when the prime in the preceding trial was visible $(M=396 \mathrm{msec})$ than when it was less easily visible $(M=$ $389 \mathrm{msec})[F(1,23)=43.90]$ and when the prime in the preceding trial was noncorresponding $(M=396 \mathrm{msec})$ rather than corresponding $(M=389 \mathrm{msec})[F(1,23)=$ 28.67]. Also, the reduction of the correspondence effect following a noncorresponding trial was stronger with a more visible prime preceding $(D=20 \mathrm{msec})$ than with a less visible prime preceding $(D=6 \mathrm{msec})[F(1,23)=$ 7.01]. This data pattern is in accordance with previous results showing that participants enter into a more cautious response mode when they have clearly identified a potential source of response conflict in the preceding trial and, particularly so, when this source actually produced a response conflict (see Kunde, 2003, for a discussion). Third, responses were $6 \mathrm{msec}$ faster when the prime-target dimension was repeated from one trial to the next $[F(1,23)=13.44]$. This dimension repetition benefit was slightly stronger with prime-target correspondence $(D=$ $9 \mathrm{msec})$ than with noncorrespondence $(D=3 \mathrm{msec})$ $[F(1,23)=9.71]$ and when the preceding trial contained a visible $(D=9 \mathrm{msec})$, rather than an invisible, prime $(D=$ $4 \mathrm{msec}$, rounded) $[F(1,23)=5.62]$.

\section{Error Rates}

The analysis of error rates revealed more accurate responses with corresponding $(M=1.5 \%)$ than with noncorresponding $(M=4.6 \%)$ primes $[F(1,23)=47.30]$. The correspondence effect was more pronounced with a prime duration of $126 \mathrm{msec}(D=5.3 \%)$ than with one of $14 \operatorname{msec}(D=1.0 \%)[F(1,23)=34.34]$. Error rates were

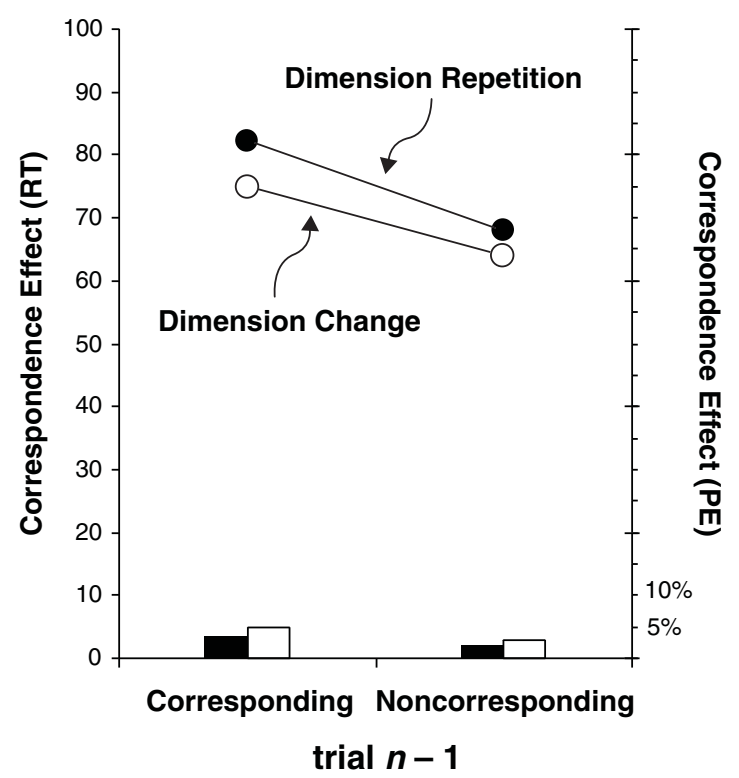

Figure 3. Experiment 1: Correspondence effects as a function of correspondence in the preceding trial. Filled symbols refer to trials in which the spatial dimension of the preceding trial was repeated; unfilled symbols refer to trials in which spatial dimensions changed. RT, response time; PE, percentage of errors. 
particularly high with a noncorresponding prime of 126msec duration, producing an overall accelerated error rate with the 126-msec prime duration $[F(1,23)=29.0]$.

Sequential effects were also present in error rates. The correspondence effect was reduced after noncorresponding trials $(D=2.5 \%)$, as compared with corresponding trials $(D=3.8 \%)[F(1,23)=5.40]$. This effect was more pronounced when the preceding prime was clearly visible (reduction from $4.2 \%$ to $1.8 \%$ ) than when it was less visible (reduction from $3.3 \%$ to $3.2 \%$ ) $[F(1,23)=12.87$ for the interaction of present correspondence, preceding correspondence, and preceding prime duration]. The error rate was slightly lower after noncorresponding trials $(M=2.7 \%)$ than after corresponding trials $(M=3.4 \%)$ $[F(1,23)=10.62]$, which likely reflects the adoption of a more conservative response mode after conflict trials. Two further effects reached significance. The reduction of the correspondence effect following noncorresponding trials was weaker with current short prime duration than with current long prime duration $[F(1,23)=8.68]$. Conceivably, this was a result of the overall lower error rate with short prime duration, leaving less room for a further reduction from a preceding response conflict. Finally, although the error rate tended to be generally lower when the prime in the preceding trial was visible, it increased slightly when the prime in the preceding trial was visible and corresponding at the same time. This produced a triple interaction of current prime duration, preceding prime duration, and preceding correspondence $[F(1,23)=8.81]$. This might reflect the adoption of a less conservative (more error prone) response mode after having perceived a prime that had benefited response selection in the previous trial.

\section{Sequential Effects With Dimension Repetition}

Mayr et al. (2003) observed sequential modulations in a 2-CRT Eriksen flanker task with response repetitions, but not with response changes. To explore whether this pattern would also hold for the present conflict task, we computed an analysis with the subset of data from conditions that resembled Mayr et al.'s conditions most closely. These were the trials in which the prime and the target were from the same dimension (either horizontal or vertical) in trial $n-1$ and trial $n$. (This is a subset of the dimension repeat trials from the previous analysis in which a repetition of prime dimension or target dimension already sufficed to count the trial as dimension repeat trial.) In half of these trials, the response was repeated, whereas in the other half the responses changed. These data were subjected to an ANOVA with the variables of correspondence in trial $n$, correspondence in trial $n-1$, and response repetition/alternation (see Table 2). The correspondence effect in RTs was reduced following noncorresponding trials $[F(1,23)=5.74]$. Although this reduction was larger when the responses were repeated $(D=11 \mathrm{msec})$ than when they changed $(D=7 \mathrm{msec})$, this apparent difference was far from significance $(F<1)$. The sequential effect was also reliable in error rates $[F(1,23)=9.61]$, but again it did not significantly differ between response changes $(D=1.4 \%)$ and repetitions $(D=3.1 \%)[F(1,23)=1.33$, $p>$.26]. Altogether, there are thus some indications of stronger sequential effects with response repetitions than with response changes, but this effect seems to be weaker than that in the version of the Eriksen flanker task used by Mayr et al. (2003).

\section{Prime Discrimination Performance}

The mean percentage of correct prime discriminations was much higher with a prime duration of $126 \mathrm{msec}(M=$ $92.1 \%)$ than with one of $14 \mathrm{msec}(M=32.0 \%)[t(23)=$ $16.4, p<.01]$. However, even with a 14-msec prime duration, performance was above the chance level of $25 \%$ $(p<.01$, one sample $t$ test). Hence, 14-msec primes were by far less visible than 126-msec primes, but they were not entirely indiscriminable.

\section{Discussion}

Experiment 1 revealed three major results. First, sequential effects occur even when the stimuli (and the responses) in subsequent trials vary on different spatial dimensions and, hence, neither the primes nor the responses are repeated from one trial to the next. This clearly shows that sequential modulations, at least in the present paradigm, cannot originate from an unbalanced proportion of incomplete $\mathrm{S}-\mathrm{R}$ repetitions in the successions of congruent and incongruent trials, as the repetition/alternation account implies. The sequential reduction of the correspondence effect was about $15 \%$ of the overall correspondence effect, which is well within the range of the sequential effects of, for instance, the Eriksen effect (e.g., Gratton

Table 2

Experiment 1: Response Times (RTs, in Milliseconds) and Error Rates (Percentages of Errors [PEs]) as a Function of Response Repetition and Correspondence in Trials $n$ and $n-1$ for Trials in Which Prime and Target Dimensions Were Identical and Repeated

\begin{tabular}{|c|c|c|c|c|c|c|c|c|}
\hline \multirow[b]{4}{*}{ Trial $n-1$ Correspondence } & \multicolumn{8}{|c|}{ Trial $n$} \\
\hline & \multicolumn{4}{|c|}{ Response Repetition } & \multicolumn{4}{|c|}{ Response Alternation } \\
\hline & \multicolumn{2}{|c|}{ Noncorresponding } & \multicolumn{2}{|c|}{ Corresponding } & \multicolumn{2}{|c|}{ Noncorresponding } & \multicolumn{2}{|c|}{ Corresponding } \\
\hline & RT & $\mathrm{PE}$ & RT & $\mathrm{PE}$ & RT & $\mathrm{PE}$ & RT & $\mathrm{PE}$ \\
\hline Noncorresponding & 415 & 2.7 & 339 & 0.6 & 429 & 4.4 & 370 & 2.2 \\
\hline Corresponding & 418 & 0.7 & 331 & 0.7 & 425 & 5.5 & 359 & 1.9 \\
\hline
\end{tabular}


et al., 1992). This result shows that some modulations of the prime-target correspondence effect are possible, but the mechanism causing these modulations does not seem to be powerful enough to remove the correspondence effect entirely.

Second, sequential effects were larger with a long prime duration than with a short prime duration in the preceding trial. Increasing the prime duration had two effects: It increased the correspondence effect (and thus, the size of the response conflict), and it improved prime perceptibility. Hence, sequential effects depend on the size and/or awareness of response conflict. This result accords quite well with a gating account, which assumes that irrelevant stimulus information becomes disconnected from the response system when it has turned out to be harmful (i.e., after a response conflict). Conceivably, such a disconnection is more likely to occur, the more detrimental the irrelevant information was, and the more discernible this detrimental impact was. Future research should clarify the relative contribution of the two (not mutually exclusive) factors of size and awareness of response conflict (Schütz \& Vorberg, 2003).

Third, when the prime and the target were from the same dimension in succeeding trials (both horizontal or both vertical), there was a tendency toward smaller sequential effects with response changes than with response repetitions. This observation is in line with similar observations from the Eriksen flanker task by Mayr et al. (2003). However, on the basis of this finding, these authors concluded that sequential effects result from an unbalanced proportion of complete prime-target repetitions (which occur in the response repetition, but not in the response alternation, case). This conclusion seems premature, since we observed robust sequential effects even when dimensions changed-hence, when neither the primes nor the targets/ responses were repeated. It therefore seems more likely that true conflict-driven modulation was occluded somehow in Mayr et al.'s analyses of within-dimension response alternations. For example, Stadler and Hogan (1996) found that RTs are unusually high for noncorresponding flankers when target and flanker items reverse from the preceding trial (e.g., $\langle<\rangle<\langle\rightarrow\rangle>\langle>\rangle$ ). In such a situation, a previously to-be-ignored stimulus has to be responded to, which renders it a subject of negative priming. In Mayr et al.'s analysis of target change trials, such an effect would inflate RTs in NC-NC trials, possibly masking an underlying conflict adjustment effect. This negative priming effect was less pronounced in our paradigm, probably because the prime and the target were not identical here but the prime was a smaller replica of the target. At any rate, our results suggest that confining the analysis to target/response alternations is not a very useful way of eliminating problems with $S-R$ repetitions. We therefore prefer to base our conclusions on the dimension change manipulation, which thoroughly removes all types of repetitions but reveals robust sequential effects nevertheless. We thus conclude that sequential effects, at least in the present paradigm, do not result from particular S-R repetitions but reflect an adaptation to response conflict.

\section{EXPERIMENT 2}

Response conflict can occur for several reasons. In Experiment 1 , such conflict resulted from primes that, in the case of noncorrespondence, signaled a different response than did the target. Another way to induce conflict is to present stimuli in locations different from response locations (i.e., presenting a stimulus on the left side that requires a right-sided response).

A general conflict adaptation mechanism should be triggered by all types of conflict, independently of the peripheral event that caused it. Some preliminary support for such a general conflict adaptation mechanism comes from brain-imaging studies. These studies have shown that conflicts in different tasks, such as the Eriksen task (Botvinick et al., 1999) or the Stroop task (Kerns et al., 2004), activate the same brain areas in the anterior cingulate cortex. Here, we tested another implication of a general response conflict adaptation mechanism. If conflict triggers adjustment independently of its stimulus-related origin, it can be expected that conflict due to one stimulus aspect (e.g., a noncorresponding prime) might affect correspondence effects that are due to a different stimulus aspect (e.g., a noncorresponding stimulus location). In other words, sequential effects might transfer between different types of $\mathrm{S}-\mathrm{R}$ correspondence.

To test this prediction, we varied independently two different types of S-R correspondence in Experiment 2 (see Figure 4). First, as in Experiment 1, the prime corresponded or did not correspond to the target (and thus, to the required response). Second, the target (and the prime) were presented in a location that was corresponding or noncorresponding with respect to the required response, as in a standard Simon task. We predicted that both types of S-R correspondence would affect performance. Hence, we expected a prime-target correspondence effect and a Simon effect. Moreover, each type of correspondence should affect the correspondence effect of the same type in the subsequent trial. Thus, a noncorresponding prime should reduce the prime-target correspondence effect in the subsequent trial, and a spatially noncorresponding stimulus location should reduce the Simon effect in the subsequent trial. More important, we asked whether sequential modulations might occur between different types of correspondence effects. In other words, we investigated whether prime-target correspondence might affect the spatial S-R correspondence effect of the subsequent trial and whether spatial S-R correspondence might affect the prime-target correspondence effect of the subsequent trial.

\section{Method}

\section{Participants}

Thirteen students ( 3 men and 10 women), 18-23 years of age, from the University of Halle-Wittenberg participated in the experiment to fulfill a course requirement.

\section{Apparatus, Stimuli, and Procedure}

The apparatus and stimuli were similar to those in Experiment 1 . We will only note the differences here. The prime duration was constant at $56 \mathrm{msec}$. In Experiment 2, only left-pointing and 


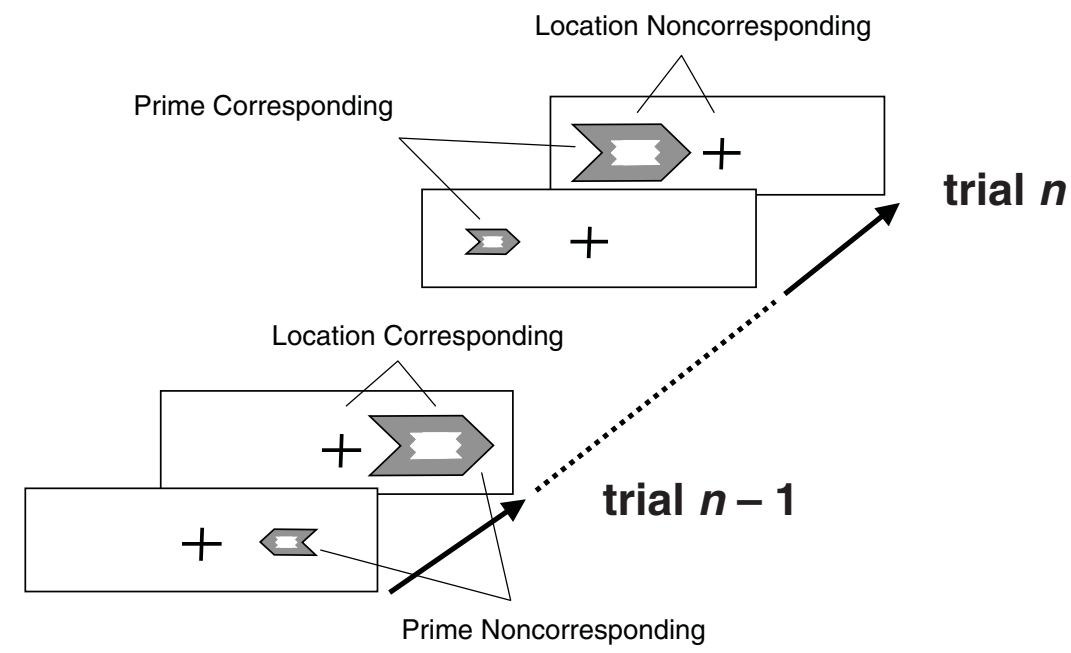

Figure 4. Sample trials in Experiment 2. In each trial, the target location was either corresponding/noncorresponding to the required response, and the prime was either corresponding/noncorresponding to the target.

right-pointing primes and arrows were used, in order to reduce the number of trials necessary to achieve an equiprobable succession of experimental trials. The stimuli were presented laterally-that is, the center of the primes and the targets was now presented $30 \mathrm{~mm}$ to the left or right of the fixation cross. Within a trial, the prime and the target always appeared at the same location (either left or right). Left-pointing targets indicated a response with the left index finger, and right-pointing targets indicated a response with the right index finger. The responses were made on two response keys that were separated by $150 \mathrm{~mm}$ and located in front of the participants. There were eight trial types, resulting from the combination of 2 primes (left-pointing or right-pointing) $\times 2$ targets (left-pointing or rightpointing) $\times 2$ locations (left or right of fixation). These eight trial types were arranged in blocks of 64 trials, so that each succession of the eight trial types occurred once. After 64 practice trials, the participants performed five blocks of 64 experimental trials (i.e., 320 experimental trials). Following the experimental trials, a prime discrimination task (with 128 trials) similar to that in Experiment 1 was administered.

\section{Results}

Trials following errors were removed from subsequent analysis. One other response was excluded, since it had an
RT longer than 1,500 msec. Mean RTs were subjected to an ANOVA with the repeated measures of present primetarget correspondence, present spatial correspondence, preceding prime-target correspondence, and preceding spatial correspondence. Table 3 shows the mean RTs from the combination of these variables.

Responding was faster with prime-target correspondence $(M=398 \mathrm{msec})$ than with prime-target noncorrespondence $(M=310 \mathrm{msec})[F(1,12)=707.94]$ and was faster with spatial correspondence $(M=348 \mathrm{msec})$ than with spatial noncorrespondence $(M=360 \mathrm{msec})$ $[F(1,12)=7.17]$. These correspondence effects interacted: The spatial correspondence effect was larger with prime-target correspondence than with prime-target noncorrespondence (or vice versa, prime target correspondence was larger with spatial correspondence than with spatial noncorrespondence) $[F(1,12)=12.63]$.

Several sequential effects were present. The size of the spatial correspondence effect and the prime-target correspondence effect as a function of preceding spatial correspondence and preceding prime-target correspondence

Table 3

Experiment 2: Response Times (RTs, in Milliseconds) and Error Rates (Percentages of Errors [PEs]) as a Function of Prime-Target Correspondence and Spatial Correspondence in Trials $n$ and $n-1$

\begin{tabular}{|c|c|c|c|c|c|c|c|c|}
\hline \multirow[b]{4}{*}{ Trial $n-1$} & \multicolumn{8}{|c|}{ Trial $n$} \\
\hline & \multicolumn{4}{|c|}{ Prime Noncorresponding } & \multicolumn{4}{|c|}{ Prime Corresponding } \\
\hline & \multicolumn{2}{|c|}{$\begin{array}{c}\text { Location } \\
\text { Noncorresponding }\end{array}$} & \multicolumn{2}{|c|}{$\begin{array}{c}\text { Location } \\
\text { Corresponding }\end{array}$} & \multicolumn{2}{|c|}{$\begin{array}{c}\text { Location } \\
\text { Noncorresponding }\end{array}$} & \multicolumn{2}{|c|}{$\begin{array}{c}\text { Location } \\
\text { Corresponding }\end{array}$} \\
\hline & RT & $\mathrm{PE}$ & $\mathrm{RT}$ & $\mathrm{PE}$ & RT & $\mathrm{PE}$ & RT & $\mathrm{PE}$ \\
\hline \multicolumn{9}{|l|}{ Prime noncorresponding } \\
\hline Location noncorresponding & 394 & 8.6 & 397 & 6.0 & 321 & 1.6 & 321 & 1.7 \\
\hline Location corresponding & 408 & 14.4 & 392 & 4.3 & 333 & 3.6 & 292 & 0.4 \\
\hline \multicolumn{9}{|l|}{ Prime corresponding } \\
\hline Location noncorresponding & 390 & 14.5 & 404 & 7.3 & 304 & 1.5 & 309 & 1.2 \\
\hline Location corresponding & 407 & 28.4 & 396 & 5.6 & 324 & 4.6 & 276 & 0.4 \\
\hline
\end{tabular}


Table 4

Experiment 2: Spatial Correspondence Effect and Prime-Target Correspondence Effect as a Function of Spatial Correspondence and Prime-Target Correspondence in the Preceding Trial

\begin{tabular}{|c|c|c|c|c|c|c|c|c|}
\hline \multirow[b]{4}{*}{ Effect } & \multicolumn{8}{|c|}{ Trial $n-1$} \\
\hline & \multicolumn{4}{|c|}{ Spatially Corresponding } & \multicolumn{4}{|c|}{ Spatially Noncorresponding } \\
\hline & \multicolumn{2}{|c|}{ Prime Corresponding } & \multicolumn{2}{|c|}{ Prime Noncorresponding } & \multicolumn{2}{|c|}{ Prime Corresponding } & \multicolumn{2}{|c|}{ Prime Noncorresponding } \\
\hline & $\Delta \mathrm{RT}$ & $\Delta \mathrm{PE}$ & $\Delta \mathrm{RT}$ & $\Delta \mathrm{PE}$ & $\Delta \mathrm{RT}$ & $\Delta \mathrm{PE}$ & $\Delta \mathrm{RT}$ & $\Delta \mathrm{PE}$ \\
\hline Spatial correspondence & 30 & 13.5 & 28 & 6.7 & -9 & 3.7 & -2 & 1.2 \\
\hline Prime-target correspondence & 101 & 14.5 & 88 & 7.4 & 91 & 9.5 & 74 & 5.6 \\
\hline
\end{tabular}

Note $-\Delta \mathrm{RT}=$ correspondence effect in response times (noncorresponding - corresponding); $\Delta \mathrm{PE}=$ correspondence effect in percentage of errors.

are listed in Table 4 and illustrated in Figure 5. First, as in Experiment 1, the prime-target correspondence effect was larger after corresponding prime-target conditions $(D=$ $96 \mathrm{msec}$ ) than after noncorresponding prime-target conditions $(D=81 \mathrm{msec})[F(1,12)=17.93]$, and responding was overall $6 \mathrm{msec}$ faster after corresponding than after noncorresponding prime-target trials $[F(1,12)=7.69]$. Second, the spatial correspondence effect was large after spatially corresponding trials $(D=28 \mathrm{msec})$ but tended to be negative after spatially noncorresponding trials $(D=$ $-6 \mathrm{msec})[F(1,12)=31.39]$.

Third, there were sequential modulations between correspondence effects: The prime-target correspondence effect was larger after spatially corresponding conditions $(D=94 \mathrm{msec})$ than after spatially noncorresponding conditions $(D=83 \mathrm{msec})[F(1,12)=11.11$; see Figure 5, left panel]. This was particularly the case when the present trial was also spatially corresponding, producing a significant prime-target correspondence $\times$ preceding spatial correspondence $\times$ current spatial correspondence interaction $[F(1,12)=6.01]$. Influences of preceding prime-target correspondence on present spatial correspondence were also present, although not in a very consistent manner in RTs (but see the error rates).

The analysis of error rates revealed more accurate responding with prime-target correspondence $(M=1.9 \%)$ than with noncorrespondence $(M=11.1 \%)[F(1,12)=$ $29.05]$ and more accurate responding with spatial correspondence $(M=3.4 \%)$ than with spatial noncorrespondence $(9.6 \%)[F(1,12)=19.08]$. These effects interacted $[F(1,12)=18.28]$, but in a manner opposite to RTs: The spatial correspondence effect was larger with prime-

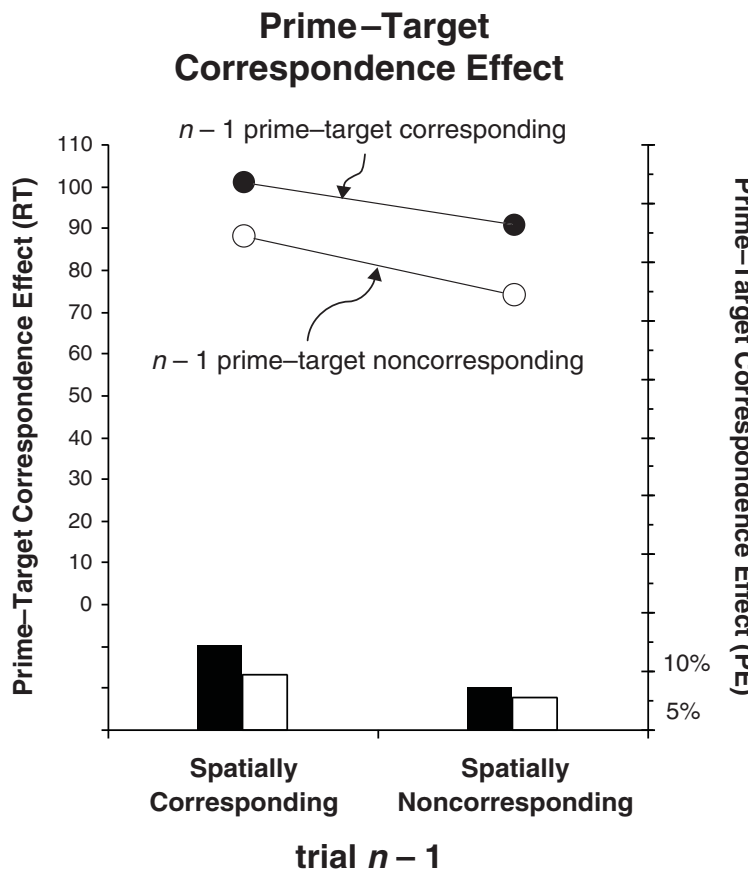

\section{Spatial Correspondence Effect}

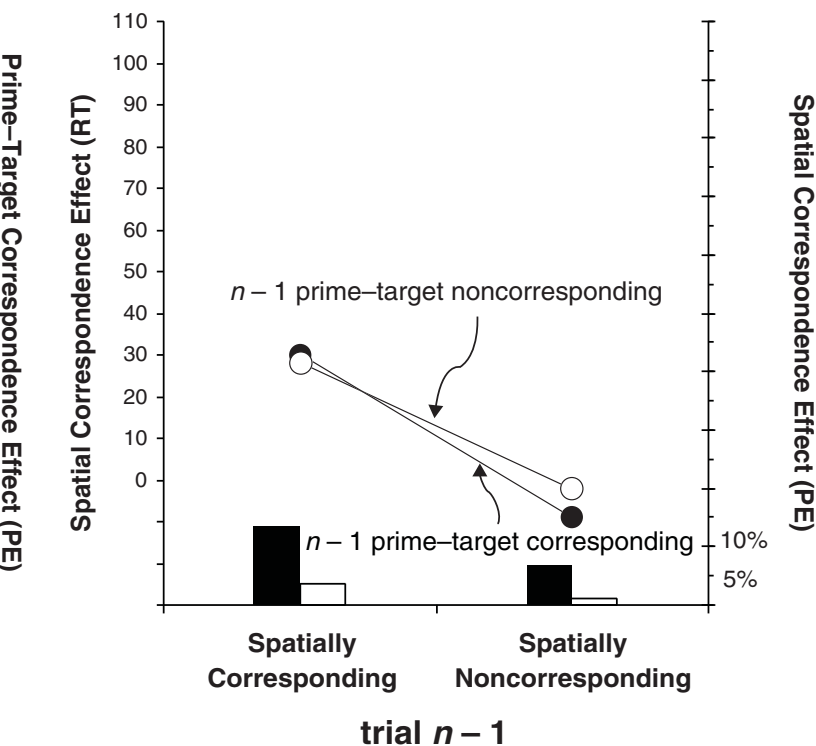

Figure 5. Experiment 2: Prime-target correspondence effect (left panel) and spatial correspondence effect (right panel) as a function of spatial correspondence in the preceding trial and prime-target correspondence in the preceding trial. Filled symbols refer to trials in which the preceding trial was prime-target corresponding; unfilled symbols refer to trials in which the preceding trial was prime-target noncorresponding. RT, response time; PE, percentage of errors. 
target noncorrespondence than with prime-target correspondence (or vice versa, prime target correspondence was larger with spatial noncorrespondence than with spatial correspondence). Due to the possibility of a speedaccuracy trade-off, we are reluctant to interpret this interaction. More important, there were sequential effects in error rates. First, the spatial correspondence effect was smaller following a spatially noncorresponding trial $(D=$ $2.5 \%)$ than following a spatially corresponding trial $(D=$ $10.1 \%)[F(1,12)=12.94]$. In addition, a spatially noncorresponding trial reduced the error rate in the following trial by $2.4 \%$, on average, in comparison with spatially corresponding trials $[F(1,12)=7.62]$. Second, the prime-target correspondence effect was smaller with a noncorresponding prime in the preceding trial $(D=6.5 \%)$ than with a corresponding prime in the preceding trial $(D=12.0 \%)$ $[F(1,12)=5.87]$. Third, there were sequential modulations between correspondence effects. The spatial correspondence effect was smaller after a noncorresponding prime-target trial $(D=3.9 \%)$ than after a corresponding prime-target trial $(\mathrm{D}=8.6 \%)[F(1,12)=4.77, p<$ $.05]$, and the prime-target correspondence effect tended to be smaller after a spatially noncorresponding trial $(D=$ $7.6 \%)$ than after a spatially corresponding trial $(D=11.0)$ $[F(1,12)=3.57, p<.085]$.

\section{Prime Discrimination}

The mean percentage of correct prime discriminations was $M=65.6 \%$. Although this is far from perfect, it is significantly above the chance level of $50 \%[t(1,12)=$ $3.25, p<.01$, one-sample $t$ test].

\section{Discussion}

In Experiment 2, each trial contained two sources of S-R correspondence: (1) prime-target correspondence and (2) spatial correspondence. Both types of correspondence affected performance. In addition, each type of correspondence affected the size of the respective correspondence effect sequentially; that is, a spatially noncorresponding $\mathrm{S}-\mathrm{R}$ event reduced the spatial correspondence effect in the subsequent trial, and a noncorresponding prime-target event reduced the prime-target correspondence effect in the subsequent trial. Most important, there were sequential modulations between correspondence effects. A spatially noncorresponding $\mathrm{S}-\mathrm{R}$ event reduced the prime-target correspondence effect in the subsequent trial (in terms of RT and PE), and a noncorresponding prime-target event reduced the spatial correspondence effect in the subsequent trial (in terms of PE). This finding is of particular interest, because it suggests that sequential effects in different paradigms have a common functional basis.

However, there is an asymmetry of the cross-type effects. The prime-target correspondence effect was modulated by both a noncorresponding stimulus location and a noncorresponding prime in the preceding trial (see the left panel in Figure 5). By contrast, the Simon effect was affected by a noncorresponding stimulus location but was affected much less (at least in RTs) by a noncorresponding prime in the preceding trial (see the right panel in Figure 5). This asymmetry might point to task-specific sequential mechanisms for the Simon effect. Basically, the data are in accordance with the idea that the prime-target correspondence effect is affected by a general conflict adaptation mechanism that is triggered by any conflict signal, whereas the Simon effect was subject to more taskspecific mechanisms. This issue will be addressed further in the General Discussion section below.

\section{GENERAL DISCUSSION}

In the present study, we investigated sequential modulations of correspondence effects. The repetition/alternation account sees these effects as an artifact from unbalanced proportions of incomplete $\mathrm{S}-\mathrm{R}$ repetitions in the trial sequences under consideration. The gating account considers them to be an adaptation to response selection problems experienced. Viewed from the perspective of research on cognitive control, the repetition/alternation account appears to be the less interesting stance-less interesting because it is based on well-known low-level mechanisms that are not normally construed as cognitive control operations. The present results suggest that such low-level processes are not the only source of sequential effects. Two observations motivate this conclusion, which we will discuss in turn.

\section{Sequential Effects Without S-R Repetitions}

Experiment 1 revealed sequential modulations even though neither stimuli nor responses were repeatedhence, in dimension change trials. This result is not easily reconciled with a repetition/alternation account. Such an account would have to assume that some kind of higher level repetition nevertheless occurred in dimension change trials. For example, one may argue that an up-pointing target arrow followed by a right-pointing target arrow can be construed as an $\mathrm{S}-\mathrm{R}$ repetition where the previous arrow is mentally rotated $90^{\circ}$ clockwise. To test for such a mental rotation strategy, we analyzed the data as a function of orientation disparity between trial $n$ and $n-1\left(90^{\circ}, 180^{\circ}\right.$, or $270^{\circ}$ ). This analysis revealed no impact of previous target orientation $\left(90^{\circ}, 413 \mathrm{msec} ; 180^{\circ}, 415 \mathrm{msec} ; 270^{\circ}\right.$, $414 \mathrm{msec} ; F=1.1, p>.33)$. It is therefore unlikely that mental rotation of stimulus displays occurred between trials. Another higher order repetition effect could be as follows: Identical prime-target pairs might be grouped together perceptually as one object looming toward the observer. The repetition of such a looming-toward event (hence, a C-C sequence) might speed up RTs, thereby resulting in a stronger correspondence effect and, thus, a sequential modulation. This explanation is hard to rule out with the present paradigm. We note, however, that no participant reported such a looming-toward impression. Moreover, this account fails to explain sequential modulations without $\mathrm{S}-\mathrm{R}$ repetitions and different stimulus material (Kerns et al., 2004; Wühr, 2005). 
An obvious question in view of our results is why Mayr et al. (2003) failed to observe sequential effects with dimension change trials in their version of the Eriksen task. One difference is quite apparent. As compared with the present study, RTs were far higher (about 60\%) and error rates far lower (about 66\%), suggesting a strong focus on accuracy. It might be that operations for the handling of response conflict come into play only when the danger of error commission is considerable-in other words, when speed pressure is high.

\section{Sequential Effects Across Types of S-R Correspondence}

The second important finding was that correspondence effects of one type (spatial correspondence) sequentially reduced correspondence effects not only of the same type, but also of the other type (prime-target correspondence). Again, this fits well with the idea of a general conflict adaptation mechanism that is triggered by different types of conflict and capable of handling different types of conflict. However, two notes of caution are warranted here.

First, both of the two types of S-R correspondence that we combined relied on spatial codes (both arrows and locations have a spatial component). This means that we showed sequential effects across tasks when both tasks were spatial in nature. What remains to be tested is whether sequential effects also transfer between spatial and nonspatial tasks. Given the observation that spatial and nonspatial conflict tasks index the same brain areas, such a transfer can reasonably be expected (e.g., Botvinick et al., 1999; Kerns et al., 2004).

Second, our data are not entirely consistent with a general conflict adaptation device. If conflict adaptation operated entirely independently of the peripheral cause of conflict (prime form or stimulus location), one should find full transfer of sequential effects from one type of correspondence to the other. This was not, however, observed. Between-effect modulations tended to be smaller than within-effect modulations (see Figure 5). In particular, the observation that spatial noncorrespondence sequentially modulated the prime-target effect, whereas prime-target correspondence did not sequentially modulate the Simon effect, suggests task-specific sources of sequential modulations of Simon-type effects. These Simon-specific effects might have something to do with partial repetitions of S-R features, which seem to have no notable impact on sequential modulations of the prime-target correspondence effect (as was seen in Experiment 1). To clarify this, it might be worth combining four-choice versions of the present tasks, which would allow the removal of all types of S-R repetitions.

Altogether, the available evidence portrays a picture suggesting more than one cause of sequential modulations of correspondence effects. One mechanism might relate to repetitions or alternations of stimuli and/or responses between subsequent $\mathrm{S}-\mathrm{R}$ episodes. This mechanism can explain sequential effects when such S-R repetitions actually occur. Another mechanism appears to monitor and regulate response conflicts. This mechanism can explain the transfer of sequential effects between spatial dimensions and tasks observed in the present study. The necessary preconditions for these mechanisms and their functional interplay remain to be scrutinized. We consider this to be a worthwhile project that would improve our understanding of how humans maintain adaptive behavior in a world full of response-eliciting stimulation.

\section{REFERENCES}

Bertelson, P., \& Renkin, A. (1966). Reaction times to new versus repeated signals in a serial task as a function of response-signal time interval. Acta Psychologica, 25, 132-136.

Botvinick, M. M., Braver, T. S., Barch, D. M., Carter, C. S., \& Cohen, J. D. (2001). Conflict monitoring and cognitive control. Psychological Review, 108, 625-652.

Botvinick, M. [M.], Nystrom, L. E., Fissell, K., Carter, C. S., \& Cohen, J. D. (1999). Conflict monitoring versus selection-for-action in anterior cingulate cortex. Nature, 402, 179-181.

Gratton, G., Coles, M. G. H., \& Donchin, E. (1992). Optimizing the use of information: Strategic control of activation of responses. Journal of Experimental Psychology: General, 121, 480-506.

Greenwald, A. G., Draine, S. C., \& Abrams, R. L. (1996). Three cognitive markers of unconscious semantic activation. Science, 273, 1699-1702.

Hommel, B., Proctor, R. W., \& Vu, K.-P. L. (2004). A featureintegration account of sequential effects in the Simon task. Psychological Research, 68, 1-17.

Kerns, J. G., Cohen, J. D., MacDonald, A. W., III, Cho, R. Y., Stenger, V. A., \& CARTER, C. S. (2004). Anterior cingulate conflict monitoring and adjustments in control. Science, 303, 1023-1026.

Kunde, W. (2003). Sequential modulations of stimulus-response correspondence effects depend on awareness of response conflict. Psychonomic Bulletin \& Review, 10, 198-205.

Kunde, W., \& StöcKer, C. (2002). A Simon effect for stimulus response duration. Quarterly Journal of Experimental Psychology, 55A, 581-592.

Mayr, U., Awh, E., \& Laurey, P. (2003). Conflict adaptation effects in the absence of executive control. Nature Neuroscience, 5, 450-452.

Neumann, O., \& Klotz, W. (1994). Motor responses to nonreportable, masked stimuli: Where is the limit of direct parameter specification? In C. Umiltà \& M. Moscovitch (Eds.), Attention and performance XV: Conscious and nonconscious information processing (pp. 123-150). Cambridge, MA: MIT Press, Bradford Books.

Norman, D. A., \& Shallice, T. (1986). Attention to action: Willed and automatic control of behavior. In R. J. Davidson, G. E. Schwartz, \& D. Shapiro (Eds.), Consciousness and self-regulation: Advances in research and theory (Vol. 4, pp. 1-18). New York: Plenum.

Praamstra, R., Kleine, B.-U., Schnitzler, A. (1999). Magnetic stimulation of the dorsal premotor cortex modulates the Simon effect. NeuroReport, 10, 3671-3674.

Reeve, T. G., \& Proctor, R. W. (1984). On the advance preparation of discrete finger responses. Journal of Experimental Psychology: Human Perception \& Performance, 10, 541-553.

Schütz, K., \& Vorberg, D. (2003). Auf der Suche nach sequentiellen Effekten in Primingaufgaben: Welche Rolle spielt der zeitliche Abstand zwischen Prime und Target? [Searching for sequential effects in priming tasks: What is the role of the temporal distance between prime and target?] In J. Golz, F. Faul, \& R. Mausfeld (Eds.), Experimentelle Psychologie: Abstracts der 45. Tagung experimentell arbeitender Psychologen (p. 137). Lengerich: Pabst.

Simon, J. R. (1969). Reactions toward the source of stimulation. Journal of Experimental Psychology, 81, 174-176.

SoETENS, E., (1998). Localizing sequential effects in serial choice reaction time with the information reduction procedure. Journal of Experimental Psychology: Human Perception \& Performance, 24, 547-568.

Stadler, M. A., \& Hogan, M. E. (1996). Varieties of positive and negative priming. Psychonomic Bulletin \& Review, 3, 87-90. 
Stürmer, B., Leuthold, H., Soetens, E., Schröter, H., \& Sommer, W. (2002). Control over location-based response activation in the Simon task: Behavioral and electrophysiological evidence. Journal of Experimental Psychology: Human Perception \& Performance, 28, 13451363.

Vorberg, D., Mattler, U., Heinecke, A., Schmidt, T., \& SchwarzBACH, J. (2003). Different time courses for visual perception and ac- tion priming. Proceedings of the National Academy of Sciences, 100, 6275-6280.

WÜHR, P. (2005). Evidence for gating of direct response activation in the Simon task. Psychonomic Bulletin \& Review, 12, 282-288.

(Manuscript received July 20, 2004; revision accepted for publication February 18, 2005.) 